\title{
Il feticcio e il visuale
}

\author{
MASSIMO CANEVACCI \\ Università di Roma I
}

\begin{abstract}
In many historical societies, things have not been so divorced from the capacity of persons to act and the power of words to communicate. That such a view of things had not disappeared even under the conditions of occidental industrial capitalism is one of the intuitions that underlay Marx's famous discussion, in Capital, of the 'fetishism of commodities'. Appadurai (1986: 4).
\end{abstract}

\section{FARSI VEDERE}

Compito di questa relazione è di esplorare le possibilità innovative per una antropologia visuale applicata alla crescente importanza della comunicazione urbana, pubblicitaria e controculturale. A tal fine si è tentato di elaborare un quadro teorico di riferimento per mettere a fuoco la "natura" particolare delle merci contemporanee: il loro essere merci-visuali con un valore aggiunto di tipo comunicativo.

Le merci visuali sono per "essenza" fantasmatiche. Purtuttavia le forme contemporanee assunte dalla fantasmagoria visuale si differenziano profondamente dalla potenza estraniante delle merci tradizionali. Per cogliere i codici delle nuove fantasmagorie è necessario ripartire dalla nozione di feticismo e adattarla ai nuovi livelli della mercificazione. E allora i feticci visuali, proliferanti dalla comunicazione ad alta tecnologia, sono a tal punto incorporati dalle nuove merci che il metodo stesso dell'osservazione ne deve tener conto. Esso si ridefinisce come osservazione osservante, in quanto colloca la globalità cognitiva dell'essere spettatore tutta dentro il frame dell'osservazione e, nello stesso tempo, tutta fuori. Questo saltare tra l'osservare e l'osservarsi - questa meta-osservazione- è l'approccio adeguato (o conforme) alla decodifica dissolutiva della comunicazione visuale.

Farsi vedere. Per dispiegare il punto di vista dell'osservazione osservante ci si deve collocare su questa pro-posizione. Una posizione sensibile non tanto alla semiotica, all'estetica, alla comunicazione, quanto all'atto "passivo" del vedere. Farsi vedere: non nel senso di mostrarsi, ma nei molti 
sensi di sviluppare qualità sensitive fondate sulle percezioni dello sguardo, sulle sensibilità del vedere, del trasformarsi oltre il soggetto-in-visione, del mutarsi in vedere, in cosa-che-vede. Farsi sguardo, farsi occhio, farsi.

Nel farsi si sottolinea un'attività trasformativa di tipo riflessivo che coinvolge il soggetto, fino alla sua mutazione in cosa-che-vede; nel vedere si concentra il processo riflessivo nell'attività polimorfa, sensibile, emozionata dello sguardo interpretante. Farsi vedere significa collocarsi nella posizione - nel punto di vista - che sta totalmente dentro i flussi visuali e, nello stesso tempo, totalmente al di fuori. Farsi vedere significa allenarsi ad osservarsi mentre si osserva. A saper saltare tra un tutto interno al "frame" della visione e un tutto esterno. Farsi vedere significa sfidare la fantasmagoria delle merci-visuali diventando "cosa" vedente, feticcio "in" visione e "della" visione.

A tal fine, la presente ricerca si basa su una metodologia che utilizza quattro indicatori concettuali: la vita sociale delle merci-visuali, la biografia culturale delle merci, le macchine biologiche, il feticismo metodologico.

Tutti e quattro questi indicatori sono articolazioni differenziate del feticismo applicato alla contemporaneità. E', infatti, attraverso la rielaborazione del feticismo che si determina la specificità di un approccio antropologico, a cui - tra l'altro- il termine appartiene per competenze. E il feticismo visuale "vede" le nuove merci sempre più come soggetti, con una loro biografia, una loro biologia, una loro vita sociale. In questa "visione" il feticismo visuale si trasforma in feticismo metodologico. In Farsi vedere. Scopo del feticismo metodologico è favorire la dissolvenza delle merci-feticcio di tipo visuale lacerando e esasperando la loro seduzione: il loro sex-appeal inorganico ${ }^{1}$.

L'antropologia della comunicazione inquadra le merci-visuali come il suo oggetto che si trasforma in soggetto biografico, in feticci biologici, ne ridefinisce il valore e tenta di pervenire attraverso le stesse trame della rappresentazione ad un critica dell'economia politico-comunicativa. Per questo il soggetto-ricercatore deve "farsin: ovvero deve innestarsi lungo un processo uguale e contrario a quello precedente e mutarsi in cosa-chevede e che-si-vede (Canevacci 1995a).

1 Tale termine, elaborato da Walter Benjamin e ripreso recentemente da Mario Perniola, si presta a ulteriori sviluppi che colgano la potenza del "condurre-a-sè" da parte delle merci visuali. Del sedurre. 


\section{IL VALORE DELLE MERCI-VISUALI}

Ora svilupperò alcuni tentativi di delineare una teoria del valore adeguata alle merci-visuali.

Nell'attuale forma delle merci non è la quantità di lavoro astratto incorporato che ci spiega il suo valore di scambio. Tale crollo induce alla fine la teoria del valore-lavoro di stampo quantitativo (e quindi oggettivistico, naturalistico, ottocentesco). Marx, infatti, riteneva che il processo produttivo stesso avrebbe reso il lavoro pura erogazione di forzalavoro, quantitativamente misurabile come una merce tra le merci.

Gli stessi classici dualismi tra lavoro vivo e lavoro morto, lavoro astratto e lavoro concreto, struttura e sovrastruttura sono del tutto inadeguati a comprendere la "natura" delle nuove merci. Il lavoro vivo postfordista è marginale rispetto al processo di valorizzazione su basi tecnoinformatiche. Mentre il lavoro astratto, invece di essere considerato pura erogazione quantitativa e indifferente di forza lavoro estraniata, può essere ripensato come forza cognitiva e progettuale trainante del sapere tecnico-scientifico e della valorizzazione. Ecco perchè il restauro del lavoro concreto diventa un'invenzione regressiva che idealizza comunità e saperi inesistenti.

Ciò è verificabile dallo sviluppo determinante della cosiddetta "cultura d'impresa" (Gagliardi 1986) —inerente la gestione dell'insieme dei processi comunicativi che legano in fitte trame simboliche le direzioni strategiche, amministrative, operative- che da tempo si è rivolta all'antropologia culturale per comprendere, modellare e trasmettere la cultura comunicativa interna: quella particolare rete di simboli e segni, codici e stili, miti e riti messi in scena durante (e oltre) l'orario di lavoro. La logica della cultura d'impresa connette organizzazione del lavoro-pubblicità-marketingvendita in un modo reticolare e immanente.

Le merci visuali contengono nel loro corpo fantasmatico un insieme articolato di flussi. Essi sono stati precisati con un approccio di tipo antropologico-economico da Arjun Appadurai, studioso di origine indiana e docente negli Usa. Questi ha delineato un modello per inquadrare le differenze dell'economia culturale contemporanea su cinque flussi in forma di panorami: technoscape, mediascape, finanscape, ideoscape, ethnoscape (1990). E in tale rete panoramatica globalizzante si costituisce la nuova forma fluttuante e disgiuntiva del potere, senza più alcun centro di tipo strutturale, per evocare vecchie terminologie semplificatrici.

In questo scenario panoramatico e fluttuante, il plusvalore relativo è solo una obsoleta metafora che non resiste alla prova della cultura d'impresa o della logica postindustriale. 
La teoria del valore-lavoro si è quindi dissolta a causa di questa incessante mutazione comunicativo-produttiva. Per questo è necessario esplorare nuovi criteri classificatori che possano essere adeguati ad una nuova critica dell'economia politico-comunicativa. Il terreno della critica si costituisce accettando la sfida dei nuovi territori del conflitto che si basano sulla triade cultura-consumo-comunicazione di cui la merce-visuale riassume e moltiplica i sensi e i valori.

\section{IL FETICISMO METODOLOGICO}

Alcuni filoni delle scienze sociali hanno accettato la sfida del mutamento, unendo la ricerca sul campo, la riflessione teorica e la critica interna alla cultura di appartenenza. E' nel districare questo triplice, interrelato livello (ricerca-teoria-critica) che è possibile ripensare il nuovo.

Abbiamo visto come la "natura" delle nuove merci si presenta come comunicazione visuale. Essa afferisce, quindi, ai territori della cultura, di una cultura in senso antropologico non più definibile come quel "complesso insieme" unitario ed omogeneo di credenze e visioni del mondo -anch'esso di matrice ottocentesca- ma come culture plurali: plurali sia all'esterno che all'interno di un determinato contesto, culture frammentarie e competitive, dissipative e decentrate, assemblate e conflittuali. Una cultura glocal: essa è, insieme, globale e locale; partecipa contemporaneamente e conflittualmente agli allargamenti globalizzanti e ai restringimenti localizzanti. Il concetto di omologazione - che atterrì ed emozionò i pubblici degli anni sessanta- non è più adeguato a cogliere la realtà di un processo complesso e conflittuale, pieno di tensioni globalizzanti e di riclassificazioni localizzanti (Canevacci 1995b).

L'anatomia di tali merci-visuali non può che essere antropologica. E le merci - come le persone- hanno una loro vita sociale, sostiene Appadurai (1986: 3). Esse non sono più "oggetti", bensì pienamente soggetti, hanno cioè una loro individualità inscritta nelle loro forme, nei loro usi, nelle loro età. Anche le merci nascono, maturano, invecchiano, si ammalano e muoiono. Hanno nomi, parentele, genealogie, evoluzioni e mutazioni, sensibilità e intelligenze. Una loro biografia. Hanno un "Corpo" pieno di simboli e segni. Sono feticci. Sono animate.

A fronte di tale potere moltiplicativo delle merci-feticcio, delle mercivisuali, il metodo dissolutivo si deve caratterizzare come un feticismo metodologico. Sempre secondo Appadurai, che per primo ha elaborato questo concetto, esso dovrebbe essere un correttivo dell'eccessivo peso affidato a una sociologia delle transazioni, che va da Marx a Marcel Mauss e giunge fino a Baudrillard. 
Even if our approach to things is conditioned necessarily by the view that things have no meanings apart from those that human transactions, attributions, and motivations endow them with, the anthropological problem is that this formal truth does not illuminate the concrete, historical circulation of things. For that we have to follow the things themselves, for their meanings are inscribed in their forms, their uses, their trajectories (Appadurai 1986: 5).

Per cui si rende necessario svolgere l'analisi delle transazioni umane lungo quelle traiettorie che vivificano le cose. "Thus, even though from a theoretical point of view humans actors encode things with significance, from a methodological point of view it is the things-in-motion that illuminate their human and social context. No social analysis of things can avoid a minimum level of what might be called methodological fetishism" (ibidem).

Nel corso del saggio, spingerò oltre questa prospettiva, in quanto il feticismo metodologico si presenta, a mio avviso, come una chiave filiforme, frastagliata e agitata, adatta a penetrare all'interno dei nuovi arcani visuali incorporati dalle nuove merci ad alto tasso di comunicazione. Si prenderà fin troppo sul serio questo concetto -forse al di là delle intenzioni di Appadurai - in quanto aderente ad un oggetto della ricerca che si è via via trasformato in soggetto, con una sua vera e propria biografia animata, una biografia-cosa, fatta di storie di vita delle cose.

Definisco feticismo metodologico quell'approccio alle forme comunicative delle cose-animate che ne dissolve il carattere mercificato attraverso lo slittamento semiotico dei codici in esse incorporate. L'interpretazione è nello stesso tempo una distruzione. E' tale distruzione ad assumere le spirali linguistiche della decostruzione. Il feticismo metodolologico è, per così dire, omeopatico. Esso cura il feticismo esasperando e dilatando le costruzioni interpretative messe in scena dalle cose stesse nel corso della loro vita comunicativa. E' animista.

Le merci hanno un corpo e un'anima. Sono dense di feticismi e animismi. Hanno un'età, una biografia, cicli vitali. Un sex-appeal, norme di attrazione e repulsione non solo per i consumatori culturali, ma anche tra loro. Certe "cose" stanno bene insieme, si suol dire: come amanti. O si sono abituate a stare insieme: come vecchi coniugi.

Ad esse si addice quello che abbiamo chiamato il metodo polifonico, ovvero la moltiplicazione dei punti di osservazione e degli stili di rappresentazione sul medesimo oggetto (Canevacci 1992). In tal modo si scopre che questo non è più qualcosa di statico che rimane immobile davanti al soggetto osservatore, ma che si è trasformato a sua volta in soggetto, un soggetto mobile che compartecipa a tutta una serie di indicatori normalmente ritenuti appartenenti alla sola sfera organica del vivente. Per questo contestualmente deve avvenire la mutazione del soggetto osservatore in cosa-che-vede. 


\section{In Farsi vedere}

Il tentativo di affrontare in una prospettiva nuova la circolazione delle merci nell'economia culturale contemporanea è vedere come le attuali forme di scambio creano valore su questo settore. La dimensione visuale crea un valore aggiunto tra il corpo della merce e il corpo del consumatore. Questo valore aggiunto vivifica nelle nuove forme del feticismo. Esso non è più una geniale metafora che dovrebbe permetterci di penetrare nell'arcano di queste merci. Le nuove merci-visuali moltiplicano con il loro "spettro" il valore delle cose.

Le dimensioni visuali delle merci non si circoscrivono solo nella stilizzazione che assumono prima di entrare nella corsa del mercato. Questo è un primo punto di crescente importanza: le merci estetizzate comunicano precisi significati multipli col loro linguaggio ventriloquo. Le merci da tempo non sono più mute (e forse non lo sono mai state del tutto), ma parlano in modo sempre più logorroico col loro stile, con lo stile in esse incorporato, che viene decodificato nel momento del consumo in modo fortemente polisemico e attivo dal consumatore glocal. Ovvero ciascun acquirente-consumatore -appartenente ai differenti contesti geografici nello scenario mondializzato del consumo culturale- riesce ad esprimere un crescente livello di sua propria interpretazione. Egli, per così dire, localizza il prodotto. Ne plasma relativamente il significato sulla base del proprio contesto. Questo adattamento localizzato della produzione culturale mondializzata decentra il senso delle cose. I linguaggi ventriloqui incorporati dalle merci-visuali si esternano, per così dire, nei vari dialetti. Anzichè piatta omologazione, tutto ciò sviluppa e accentua una tensione irriducibile tra mondializzazione e localizzazione.

In questa impostazione basata sull'economia culturale (che si colloca sulla scia inaugurata dalle tesi sull'industria culturale, seppur con prospettive profondamente mutate) si rimescolano e intrecciano, con fili-patchwork tanto di refe e che via cavo, produzione e cultura. Compito specifico attuale è quello di assumere la cultura produttivo-comunicativa come luogo centrale della ricerca, adottando per la sua decodifica metodologie, concetti, paradigmi e forme della rappresentazione ad essa adeguati. E allora una merce visuale è nello stesso tempo una pubblicità, un intermediario culturale, una soap-opera, un video-clip, la curva-sud.

Quindi, le dimensioni visuali delle merci sono sia quelle emanate dalle forme estetizzate e stilizzate delle merci (design, packaging), cioè inscritte nel loro corpo dall'ideazione alla produzione; sia quelle comunicate dalla circolazione (pubblicità), dallo scambio (una carta di credito) e, ovviamente, 
dal consumo (la merce nel suo regno: lo shopping center). Tutti questi livelli sono inscritti nei flussi simbolicamente e valorialmente produttivi che stanno avendo accelerazioni imprevedibili col perfezionamento del nuovo livello comunicato imposto dalle tecnologie interattive nei postmedia (computer, modem, cd-rom, realtà virtuale). L'insieme di tutti questi livelli costituisce la comunicazione visuale.

\section{LA BIOGRAFIA CULTURALE DELLE MERCI}

Seguendo tale approccio innovativo di antropologia economico-culturale, la produzione di merci sviluppa anche un processo cognitivo, per cui queste merci non sono più comprensibili solo come cose, ma in un mutamento significativo che penetra dentro la loro stessa "natura" simbolica (Kopytoff 1986: 64). All'interno del pensiero occidentale si è prodotta una polarità oppositiva ideal-tipica tra l'universo delle persone (che partecipano all'umanità in modo singolarizzante) e l'universo delle merci (basato su una dimensione spersonalizzata, omologata grazie al potere livellatore dell'equivalente). Tale dicotomia "umanistica" tra persona come soggetto e merce come oggetto non funziona più.

Se l'eterogeneità caratterizza le cose singolari (gli individui sono tutti diversi tra loro: sono "Singular, unique, and unexchangeable" [p. 69]), al contrario l'omogeneità dovrebbe determinare le merci equivalenti, che sono tra loro sempre identiche. Ma le merci possono non essere più solo "cose" quando i termini di valutazione siano del tutto eterogenei.

La ricerca condotta da Kopytoff ha dimostrato come, attraverso un'operazione di costanti classificazioni e riclassificazioni, le diverse culture del mondo mutano i termini con cui l'eterogeneo si trasforma in omogeneo, per diventare oggetto di scambio secondo modi tra $i$ più vari. E viceversa: si assiste ad una sacralizzazione delle cose trasformate in soggetti singolari, che non possono subire transizioni economiche perchè senza valore (parchi naturali, monumenti, opere d'arte, simboli politici, religiosi, eccetera).

Da tale premessa discende che - se le storie di vita appartengono ad un tipo di approccio delle scienze sociali che, almeno finora, ha trattato solo individui nella loro assoluta qualità di universale singolare- ora tale approccio biografico si può estendere alle merci. Secondo Kopytoff —rovesciando tali posizioni umanocentriche - i medesimi problemi culturali possono essere sollevati concentrando la ricerca "at biographies of things" (p. 66).

Per capire tali biografie si deve rispondere a problemi in gran parte analoghi a quelli riguardanti le persone: quali sono sociologicamente le 
possibilità biografiche inerenti al loro status - da dove vengono le merci, chi le ha prodotte- quale potrà essere "an ideal carreer for such things" —quali sono le età riconosciute alle merci e il loro ciclo vitale- quali sono i segni culturali inscritti in esse - come cambia il loro uso con il mutamento della loro età- cosa avviene quando si riconosce loro una mancanza di utilità finale. Così, sulla base della sua personale esperienza sul campo, Kopytoff afferma che "the biography of a car in Africa would reveal a wealth of cultural data" (p. 67): il modo del suo acquisto, come il danaro per tale scambio è stato ottenuto, la relazione tra il venditore e l'acquirente, l'uso quotidiano dell'auto, l'identità dei suoi passeggeri più frequenti, i rapporti eventuali con garage e meccanici, l'alimentazione, i passaggi di proprietà, il prezzo iniziale e quelli di seconda mano, cosa ne rimane dopo che, col passare degli anni, l'auto collassa e il suo ciclo vitale si chiude nella fase terminale. "All of these details would reveal an entirely different biography from that of a middle-class American, or Navajo, or French peasant car" (ibidem). Infine, un'auto che superi indenne il ciclo dei trent'anni -almeno così è stato calcolato sulla base di un passaggio generazionale- penetra nella categoria delle "antichità" e il suo valore inizia ad aumentare.

Nello stabilire tali inquetanti analogie tra individui e merci, così conclude Kopytoff: "In the homogenized world of commodities, an aventful biography of a thing bocomes the story of the various singulatirizations of it, of classifications and reclassifications in an uncertain worlds of categories whose importance shifts with every minor change in the context. As with persons, the drama here lies in the uncertainties of valuation and of identity" (p. 90).

Le merci, come gli uomini hanno cicli di vita, problemi di identità, modelli classificatori: la trama che le differenzia dagli umani si fa sempre più sottile e mutevole.

\section{LA MACCHINA BIOLOGICA}

E proprio sulle identità dell merci si è indirizzata la sociologa tedesca Knorr Cetina, che ha svolto un'altra ricerca originale sulle "Condizioni psicologiche e idiosincrasie comportamentali di un detector" - frutto di sei anni di osservazione partecipante (secondo le sue parole) presso il Cern, per cogliere i modelli epistemici incorporati nelle più avanzate istituzioni scientifiche. Ciò che è emerso, tra l'altro, sono le metafore utilizzate dai fisici nucleari che lavorano a Ginevra per riclassificare questi oggetti ad altissima tecnologia (trenta miglia di diametro per un detector, per sette piani sotterranei, con diecimila yards di fili via cavo, eccetera). 
Ebbene, non solo a livello del linguaggio quotidiano, ma nei veri e propri comportamenti degli scienziati e, si può dire, delle macchine stesse, si è sviluppato un linguaggio con cui ci si rivolge al detector non tanto come ad una macchina, quanto come ad un "organismo biologico con una vita e un tempo di vita loro proprio" (1994: 11). Le categorie utilizzate sono di tipo psicologico e si riferiscono alle qualità di un organismo vivente: "they suggest an autonomous being which, in the aggregate, can also be a social being" (ibidem). E' interessante osservare come la tassonomia utilizzata tratti questi detector come veri e propri individui (quindi non omologabili nè indifferenziati sulla base della legge dell'equivalente proprio come aveva sostenuto Kopytoff, ma unique and unexchangeable), con le loro idiosincrasie comportamentali e dimensioni psicologiche: queste macchine supercostose e superperfette assumono uno status bionaturale. Nello schema della Knorr Cetina, che qui riassumiamo, un detector vive una condizione che può essere, di volta in volta, definita come quella di un essere vivo-morto-ucciso-cieco-confuso, eccetera. Una età caratterizzata da quanto vive, quando diventa vecchio, se è troppo giovane, o è necessario acceleragli l'età, e così via. Esso/egli ha un corpo con possibili malattie da diagnosticare e curare. Infine, ogni detector è diverso dall'altro, ha un suo background, può o no saper simulare, è più o meno intelligente, sensibile, simpatico, eccetera.

A fronte di questa ricerca importantissima, la spiegazione offerta dalla sociologa appare discutibile: in queste classificazioni si plasmarebbe la capacità da parte dei membri dell'istituzione scientifica di avere concrete interazioni tra loro e tra loro e le cose come alterità. Forse una direzione più interessante avrebbe utilizzato proprio le categorie del neo-feticismo e del neoanimismo. Ma quello che importa qui è altro: è la diffusa sensazione tra ricercatori in esplorazione su territori innovativi che le merci - una semplice automobile o un complesso detector - abbiano una soggettività crescente, un corpo che comunica, parla, dialoga con chi le usa. Qualcosa di più che semplici giochi di metafore.

\section{LA FANTASMAGORIA VISUALE}

Il problema, allora, si presenta sul come connettere i quattro livelli appena presentati: il feticismo metodologico — che un po' tutti li riassume- si dispiega nel suo farsi vedere, con cui tenta di dissolvere le nuove, accese fantasmagorie feticiste nel corso stesso della loro rappresentazione.

L'osservazione osservante non solo spinge il soggetto ricercatore a farsi vedere, a mutarsi in cosa-che-vede, ma assiste a queste merci-visuali 
come trasfigurate in precise biografie individuali, con un sistema di codici condiviso che ne attesta l'appartenenza a un ciclo vitale biologico, con una cultura e un valore che si vivificano nella circolazione comunicativa lungo l'intero ciclo vitale delle merci stesse.

Come è noto, già Benjamin aveva avvertito nelle Esposizioni Universali della metà Ottocento la trasfigurazione non solo della merce singola, quanto della merce generale in fantasmagoria. Essa cattura la coscienza operaia non in quanto si presenta come potenza estranea, ma, al contrario, proprio in quanto penetra, seduce e cattura la coscienza di classe e la trascina nei regni delle fantasmagorie feticiste. Cioè, tradotto in termini antropologici, proprio in quanto la merce universale si presenta come la cosa più familiare disponibile. Nel rovesciamento dell'estraneazione in familiarizzazione, si compie il primo vero capovolgimento delle merci e la loro intronazione visuale in forma di esposizione.

Partendo dal fatto che il nuovo feticismo della merce -inter-connessa nei canali della comunicazione visuale dell'economia culturale- sta nel suo essere familiare, di conseguenza, come per ogni buona ricerca antropologica, si tratta di trasformare questo familiare in straniero. E' necessario, cioè, compiere un lavoro esattamente opposto a quello dei tempi di Marx: osservare le merci-visuali come estranee, a causa -paradossalmente- proprio del loro eccesso di familiarità. Nel rendere estranee le merci visuali, le si deve rappresentare come se si vedessero per la prima volta: con la stessa curiosità esotica o ingenuità infantile. Se il feticismo della merce è la cosa più familiare nella fase della comunicazione visuale, esso deve "farsi vedere" come la cosa più estranea. Ciò vale per la notissima pubblicità come per il grande film d'autore. Per Pasolini o Croneberg come per il profumo Egoiste o i jeans 501. Nel processo dissolutivo di trasformare le merci visuali da familiari a straniere si può compiere la loro (e la nostra) dereificazione.

Il feticismo compatto, che si coagula all'interno della merce-visuale e che incanta il suo consumatore, può dissolversi attraverso la narrazione stupita della sua fatticità. E tale narrazione deve "giocare" - cioè rischiare- nel campo delle strategie comunicative simboliche: penetrare dentro i codici, disvelarne le carni e le anime interiori, raccogliere le biografie delle merci-visuali. Finalmente il feticismo può farsi vedere.

A tal fine, oltre a queste strategie benjaminiane, applicheremo quelle prospettive ecologico-mentali che Bateson aveva applicato alle patologie della comunicazione e che non casualmente furono influenzate dall'animismo e dal totemismo da lui sperimentati sul campo di ricerca. Per questo il primo capitolo sarà dedicato al maestro dell'ecologia della mente, ma per strapparlo dall'uso agiografico degli ambientalisti attuali, e per 
inserirlo nelle sue specifiche competenze - storiche ed etnografiche- di sperimentatore verso nuove forme della rappresentazione, sia scritte che visuali. Animismi, feticismi, totemismi verranno così tolti dai loro contesti etnografici e inseriti nelle nuove forme della comunicazione visuale.

Nella seconda parte, presenterò tre ricerche che hanno utilizzato l'antropologia della comunicazione visuale (Canevacci 1995a) come metodo per interpretare il nesso feticista tra il corpo animale di un'auto, i corpi dei muri berlinesi, i corpi inorganici degli autocostruttori.

\section{PARTE. ELEMENTI DI EMPIRIA VISUALE}

\section{Il CORPO DELLA Tigra}

Ora vorrei mostrare come un'azienda dell'auto costruisce questa strategia comunicativa che animizza e soggettivizza la merce attraverso il nesso pubblicità-marketing-vendita.

All'interno di un rettangolo di un giallo ocra forte, giace la nuova auto della Opel, collocata su una strada impossibile e feroce, su uno sfondo come tratto da una cava di lavagna. Da un tale scenario, irto di pietre verticali, schegge aguzze, lastre taglienti e illuminato dall'ocra computerizzato si stacca il blu dell'auto. Un scritta dice: "Chi ha paura di Opel Tigra? Chi non ride mai". E' importante sottolineare il "di" - e non "della" - per comunicare un qualcosa di soggettivo, dotato di corpo e anima, di biografia. Opel Tigra è persona, è merce dalla vita sociale e comunicativa. Cognome: Opel, nome: Tigra.

Ovviamente la cosa più eloquente sta nel disegno del coupé: il suo valore aggiunto è situato cioè nella sua merce-visuale, nel suo stile dalla carica estetizzante forte e innovativa. Il paraurti sembra un muso che punta la preda prima di compiere il balzo in avanti. Il finestrino disegna due occhi animaleschi, obliqui, felini, adeguati al soggetto merce e al soggetto consumatore. E' un finestrino-fumetto. Due finestrini-occhi-di-tigra. Tutto il disegno della porta intorno al finestrino-occhio esalta uno sguardo dalla felinità obliqua. In basso si incurva una bocca spalancata: i quattro cerchi in lega dentale (senza la borchia che copre e nasconde) delle ruote sottolineano ciascuno la potenza di cinque zanne, più che semplici raggiere. Esse trasfigurano la mediazione tra autista e terreno in una vera e propria morsa. Le ruote come mascelle. Il retro-parabrezza è nero fumo e rigato, tale da accentuarne "l'animalizzazione", quasi una criniera. Lo spoiler finale è aguzzo con due punti rossi alle estremità (i fari di posizione); il paraurti ondeggia come fosse "pelo" scosso dal vento. La silouette 
globale è più alta alla coda che sul muso, accentuando la propensione allo slancio.

Tutta questa potenza visuale della merce-auto si completa con una ulteriore forzatura linguistica: Tigra, appunto, che nomina la merce-animale, la individualizza. "Tigra: scatto e potenza da felino di razza, ma docile e remissiva con il domatore", dice il depliant pubblicitario. Già negli anni Sessanta lo slogan "metti un tigre nel motore" denotava l'irrompere di un essere maschile tigrato dentro il corpo dell'auto e, di conseguenza, all'interno del sè di chi la guidava. Ora la mutazione torna al femminile forzando le gabbie del linguaggio: da un tigre alla tigra. Anzi decade l'articolo determinativo "la" che ancora oggettiva: Tigra e basta. O meglio - Opel di cognome e Tigra di nome. Questa la genealogia, questa la sua carta di identità. La visione pubblicitaria "olista" - tra codice iconico, codice verbale scritto, codice merce- produce un isomorfismo compatto quanto il suo potenziale target. La merce-auto comunica con tutta la potenza visuale dei suoi molti linguaggi: essa estetizza in modo aggressivo il suo stile (pur stemperato nel sorriso). La merce-auto è ora merce-visuale, con una sua vita, un corpo e un'anima, pronta per il mercato e la sua biografia. In tutto questo racconto visuale vi è il valore.

Sembra che i pubblicitari contemporanei abbiano preso molto sul serio l'ecologia della mente di Gregory Bateson (1976): in questa immagine, tratta da un quotidiano, è facile osservare una vera e propria trama di codici che connette il mondo minerale, con uno sfondo "selvaggio" appena stemperato in una strada sasso-dentata, il mondo animale, dalle tigri mutanti pronte a balzare in avanti, il mondo meccanico, fatto di una merce che si presenta come un grande feticcio, e il mondo umano, segmentato in possibili acquirenti che, impossessandosi delle chiavi dell'auto, si trasformano animisticamente e feticisticamente (cioè ecologicamente) in roccia aguzza, bestia feroce, mostro meccanico ... e impiegato modello. Autista e domatore.

\section{IL CORPO DEI MURI}

La città di Berlino ha molti landscape. Questo termine, secondo alcyne ricerche innovative applicate ai panorami visuali metropolitani, "has recentely emerged from a long periodo of reification to become a potent tool of cultural analysis" (Zukin 1991: 16). Ogni panorama urbano implica un preciso punto di vista $\mathrm{e}$, in particolare, "power institution have a preminent capacity to impose their view on the landscapen. In una prospettiva panoramatica, le relazioni dentro la metropoli non sono obiettiva- 
mente date, per cui dovrebbero sempre essere identiche da ogni angolo visuale, bensì sono prospettive interne costruite dalle specifiche condizioni storico-linguistiche e politico-comunicative (Appadurai 1990: 296).

La ristrutturazione della vecchia Berlino uscita dalla guerra fredda emerge come un conflitto potente tra punti di vista divergenti sul senso da dare alla nuova metropoli. Il suo vecchio centro - che si era trasformato, ai tempi del muro, in periferie di due città tra loro nemiche- ora si sta modellando in centro di una nuova metropoli. Un nuovo potente landscape con un suo nuovo visualscape. Questo è il luogo mobile del conflitto politico-comunicativo. Dei nuovi territori dell'impolitico. L'attuale politica culturale del comune ha spinto e spinge verso visioni architettoniche restaurative e apologetiche. Molti edifici centrali dal cemento prestampato, frutto-del-male coltivato dal realismo socio-geometrico ex-DDR, verranno fatti saltare in aria. Altri, invece, verranno clonati da architetti replicanti, per non turbare la coesistenza pacifico-urbanistica col passato da dimenticare o conciliare. Il panorama metropolitano berlinese vincente si configura sotto il doppio segno della lotta contro la sperimentazione e per la pratica della conformazione. Passato e presente si alleano sotto il segno dell'ordine, delle simmetrie, del previsto. Un landscape di luoghi pesanti e immobili. Architetture come ricalco senza fughe.

Il potere a Berlino si autocostruisce come nuovo landscape che celebra l'immutabilità nel momento stesso in cui sembra voler ricostruire tutto.

La struggente presenza deformante dei palazzi ex-est nella mitte, una volta ristrutturati, imbiancati e riconsegnati ai proprietari pre-'45, sembrano suggerre una medicalizzazione del quartiere più irrequieto e alternativo post'89. Una sua chirurgizzazione architetonica e sociologica. Simmetricamente Kreuznerg - da quartiere periferico di opposizione e multi-culti nella exovest- è ritornato ad essere zona centrale che attrae la speculazione edilizia contro ogni speculazione intellettuale.

Ancora per poco, la ferita non rimarginata rimane in tutta la sua visionarietà apocalittica nel Tachless, edificio autopreservatosi intatto dopo i bombardamenti, intatto e affettato da bombe chirurgiche, esposto sul nulla del suo lato posteriore dissoltosi nel nulla nel ' 45 e trasformato in terra di nessuno, spiazzo sterrato, zona morta come una casa Usher. Ora questo stesso Tachless è, forse ancora per poco, un centro sociale aperto a tutte le sperimentazioni linguistiche, forse recentemente un po' stanco, ma che deve essre difeso contro i prevedibili assalti restrutturatore e normalizzatore dei nuovi poteri urbani. Grazie al Tachless, la Berlino unificata non è tutta apologetica, ma attesta la irrinunciabilità al conflitto comunicativo, all'invenzione dei codici dissonanti, alla comunicazione visuale dura, al giocodei-corpi mobili e delle tecnologie in transito. Corpi, macchine, muri tran- 
sitivi lungo un edificio che i bombardamenti hanno lasciato aperto come un corpo steso su un tavolo chirurgico e lì dimenticato. Eppure, potenza del postmoderno, la sua visita è da tempo consigliata per un sano giro di notte con brividi alternativi controllati, dopo aver visitato musei e mostre. Il Tachless come theme-park berlinese.

Per svolgere una ricerca qualitativa sulla comunicazione visuale in questa metropoli, ho selezionato i muri di Berlino, ovvero i lati ciechi di quegli edifici - tantissimi- rimasti senza il sostegno fraterno dei palazzi contigui ormai scomparsi e mai più tornati.

Definisco il progetto che sta disegnando la Berlino post-'89 come murare il muro. Un numero enorme di edifici ex-est, infatti, viene ristrutturato nell'impossibilità di ristabilire la consecutività armonica precedente a bombardamenti e sventramenti. Una parete rimane sempre fuori. E questa parete senza più appoggio, priva di un altro edificio cui connettersi, si innalza in tutta la sua grezza rugosità di muro cieco e senza senso: è proprio in questa deprivazione di senso - di un senso funzionalmente architettonico- che essa ne acquista uno nuovo. Un senso mutilato e corrugato. Un muro cicatrice. Sono muri alti, lunghi, inscuriti dal tempo, dai molti tempi dolorosi e foschi; in genere senza alcuna finestra o col suo fantasma murato. L'impronta murata di una finestra. Muri inutili. Muri murati.

A volte qualche indisciplinato inquilino li ha forati e un'unica piccola finestrina sghemba si apre lungo l'enorme parete, un segno di disordine e di asimmetrie utile solo, almeno così si immagina, per il panorama di chi l'abita. In altri caso compaiono minuscole feritoie aperte su un muro come a difesa contro un nemico invisibile ma potenzialmente presente. Il muro diventa come un bastione medioevale che spia il panorama cirsostante e che si ritira, si contrae da questo panorama. Tanti di questi muri sono rimasti come erano, cioè crudi, con le linee di mattoni visibili, come irritati per l'improvvisa partenza del vicino. Mattoni rugosi e corrugati.

\section{Mattoni abbandonati}

Altre volte -e sono i casi più impressionanti- lungo il muro è rimasta come l'impronta della casa precedente, specie quando più piccolina: essa sopravvive là come un calco. Un'impronta. La forma è fuggita, sparita. Le linee spioventi del tetto che non-c'è-più sembrano le foto sbiadite di un caro estinto che rimangono tristemente su una tomba. Qui, una volta, al suo fianco si appoggiava un'altra casa, più piccola e che poi, per motivi incomprensibili o ormai dimenticati, se n'è andata. Per sempre. Guardan- 
do con più attenzione, è possibile decifrare i tanti glifi rimasti incastonati in questi vecchi muri, in questi muri murati, codici di un qualcosa di opaco e di terribile come una verità che si fa di tutto per scordare, per soffocare, per ricacciare nell'oblio dell'indistinto.

In un caso permane, sbiadita e démodé, la pubblicità di Neue Zeit - Der Christlich Demokratischen Union Deutschlands- il giornale "nuovo" ex-DDR che disegna in un corsivo murale il fascino dell'antico. Ed è proprio questa ambiguità a colpire e a sedurre, non di certo per nostalgie restaurative, bensì per la forza emotiva e auratica di una pubblicità sull'inesistente.

Sullo svanito. Una pubblicità sopravvissuta. Come un barbone o un clandestino albanese. In un altro, invece, compaiono i colori forti e elettrici della controcultura hip hop, i graffiti graffianti che narrano storie di militari che si masturbano sulla città oppure che comunicano la firma dell'autore: ZAK.

\section{Muri come tele o come biglietti da visita}

E' come se il vecchio muro di Berlino, ormai quasi del tutto dissolto, si fosse spostato in verticale sui lati più abbandonati di quei palazzi reclusi a testimoniare l'avvenuta ferita. Questi muri murati sono delle cicatrici. Sono muri di carne cicatrizzata, di carne scoperta e lasciata seccare all'aperto, così, oscenamente, senza possibilità di coprire la vergogna. $O$ il dolore. Muri di cicatrice. Cicatrici murate. E dopo di loro, il vuoto. Quello spazio, una volta riempito dal suo vicino - l'edificio scomparso, distrutto, eliminato con un gesto- sembra per sempre impossibilitato ad essere di nuovo pieno.

\section{E' un vuoto che si deve murare}

Ma il progetto comunale è preciso come un bisturi. A queste rughe sporche, scure, cicatrizzate, si deve rifare il lifting: ripulite e imbiancate, si è cercato di far sparire per sempre quel senso di carne morta. A volte questi muri si presentano alla sguardo panoramatico assemblati uno a fianco dell'altro, tutti lisci e abbelliti, costruendo un montaggio prospettico singolare: tutto appare un falso nuovo, simile ad un viso di una donna (o di un uomo) anziana troppo truccata. Muri truccati. Senza finestre o segni di vita, senso un segno. 
I nuovi muri di Berlino sono bianchi come fantasmi e si aggirano, da fermi lungo la mitte per ritrovare i loro compagni perduti. Tre lati sembrano ritornati normali, come se non fosse accaduto nulla per secoli in questo vecchio centro berlinese. Ma se si gira e si prendono questi palazzi alle spalle, come di sorpresa, si svela questo imbarazzante nulla. Questo quarto lato dal muro vuoto e murato. Improvvisamente l'intero orizzonte della metropoli pare coincidere con queste facce bianche e vuote: solo esse sono presenti nel panorama visuale. Un landscape ordinato e senza volto, senza senso e senza sensi.

Un panorama murato.

\section{CORPI INORGANICI}

In un noto centro sociale autogestito di Bologna, un gruppo controculturale - gli Autocostruttori- ha elaborato alcune sperimentazioni significative per un discorso che vede progressivi avvicinamenti tra corpi e muri, organico e inorganico, paesaggi di carne e panorami visuali. Nel 1996 essi hanno svolto una performance in una estrema periferia di Roma, Tor Bella Monaca, dove -come è loro solito- hanno raccolto oggetti tecnologici abbandonati lungo le strade per riassemblarli secondo un nuovo ordine e infine produrre un'opera d'arte ludica e decontestualizzata. Una sorta di deriva per elettrodomestici abbandonati, macchine senza più valori di scambio nè valori d'uso, tecnologie perse come cani abbandonati.

Questi autocostruttori praticano una estetizzazione di cose reiette, scartate, abbandonate. Di merci morte. Attraverso il taglio e il montaggio, esse tornano a vivere nuove biologie e nuove biografie come alterità aliene e disturbanti. In tal modo, apparecchi-TV-morti, scheletri di merci, tornano a vivere nuove soggettivita impreviste attraverso rivestimenti di acciaio arrotolato.

Il ferro è anche incorporato attraverso il piercing: in ogni parte dei loro corpi. Il metallo connette l'organico con l'inorganico, costruendo corpi nuovi, bodyscape in cui la differenza tra l'artificiale e il naturale appartiene ad archeologie anatomiche. Gli Autocostruttori autocostruiscono anche i loro corpi come nuovi panorami visuali. Le loro musiche sono industrial, emesse da strumenti estratti da catene di montaggio inesistenti, come da officine di un Vulcano-toyotista; settano tecnologie comunicative a circuito chiuso tipo TV-monitoring che diffondono in diretta piercing effettuati nelle zone più impensate, tatooing, scarificazioni. Il loro corpo è autocostruito in modo affine all'assemblaggio delle loro macchine biologiche: il risultato è uno stato alterato delle merci isomorfo allo stato alterato del corpo. 


\section{Entrambi sono mutoidi}

Questi autocostruttori-autocostruiti realizzano collages inaspettati attraverso elementi incongrui che scorrono dall'erotico all'esotico. Alcuni manichini femminili sono sezionati e incapsulati in un furgone come cadaveri viventi; un montaggio di pezzi di corpi e pezzi di furgone, cadaveri incorporati dal furgone come dopo un incidente stradale, un puzzle di umano, da falso-umano, di quasi-umano, di non-ancora-umano, un corpocosa che corre ripena di cadaveri mutilati. Tali cadaveri-manichini (manikinscorpse) sono seduttivi, sono un risultato in mutazione prodotto da un sex appeal inorganico. Come per il fotografo estremo Andrea Serrano - che ha realizzato un portfolio sulla Morgue, una sensuale Morgue, dove cadaveri inorganici sono erotizzati.

Si assiste ad una continuità metonimica tra gli autocostruttori che siedono sopra e dentro il veicolo, il furgone stesso e i manichini sezionati e cadaverizzati: è una catena semiotica estetizzante. Attraverso questo slittamento dei codici, ogni borderline tra il soggetto e l'oggetto, il vivente e il morto, il corpo (body) e il cadavere (corpse), l'organico e l'inorganico, la bio-logy e la iron-logy appare come una archeologia linguistica.

Finalmente, il furgone vivente può continuare la sua corsa-performance lungo la periferia di Tor Bella Monaca. Un furgone estetizzato pieno di esseri assemblati: contemporaneamente tutti vivi e tutti morti. I loro sono corpi mutoidi che muovono contro l'ordine vigente dell'estetica.

\section{BIBLIOGRAFIA}

Appadurai, A., 1986: The Social Life of Things, Cambridge: Cambridge University Press. - 1990: "Disjunture and Difference in the Global Cultural Economy", en M. Featherstone (a cura di), Global Culture, London: Sage.

BAteson, G., 1976: Verso un'ecologia della mente, Milano: Adelphi.

CANEVACCI, M., 1992: La città polifonica, Roma: SEAM.

- 1995a: Antropologia della comunicazione visuale, Genova: Costa \& Nolan.

- 1995b: Sincretismi, Genova: Costa \& Nolan.

GAGLIARDI, P. (org.), 1986: La imprese come culture, Torino: Isedi.

KNORR CETINA, K., 1994: "Primitive Classification and Postmodernity: toward a Notion of Fiction", Theory, Culture E Society, 11.

KopyTOFF, I., 1986: "The Cultural Biography of Things: Commodification as Process", en Appadurai (1986).

PERNIOla, M., 1995: Il sex appeal dell'inorganico, Torino: Einaudi.

Zukin, S., 1991: Landscape of Power. From Detroit to Disney World, Berkeley: University of California Press. 
En este ensayo se aplica el fetichismo metodológico a los nuevos "bienes-visuales" y a la "biografía" de los bienes. La mirada etnográfica permite analizar tres aspectos de la comunicación visual contemporánea: el cuerpo de un automóvil (Opel-Tigra), el cuerpo de un muro (el de Berlín), el cuerpo inorgánico (una contracultura metropolitana). El objetivo es la disolución de los fetiches visuales.

The author illustrates the application of methodological fetishism to the new "visual goods" and to the "biography" of wealth. Three aspects of current visual communication can be subject to ethnographic scrutiny: the bodywork of a car (of an Opel-Tigra, for example), a wall (such as the Berlin Wall) and an inorganic body (such as a metropolitan counterculture). The author aims at the dissolution of these visual fetishes. 